() Н.А. Петунина, И.В. Трухин, Л.В. Трухина, Ж.М. Сизова, В.Л. Захарова

Первый Московский государственный медицинский университет имени И.М. Сеченова (Сеченовский университет), Москва

Сахарный диабет (СД) и сердечная недостаточность (СН) являются распространенными коморбидными состояниями, между которыми прослеживается двунаправленная взаимосвязь. Прогноз у пациентов с СН хуже, если у них есть СД, а пациенты с СД подвержены большему риску развития СН. Следует отметить, что СН четко не вписывается как в группу микрососудистых, так и в группу макрососудистых осложнений СД. Известно, что основными причинами развития СН у больных СД являются ишемическая болезнь сердца и артериальная гипертензия, однако было показано, что СД непосредственно приводит к поражению сердца с развитием так называемой диабетической кардиомиопатии, состоянию, при котором может наблюдаться как рестриктивный, так и дилатационный фенотипы, патогенез каждого из которых подробно освещается в публикации. В то время как все медикаментозные и немедикаментозные виды лечения СН одинаково эффективны вне зависимости от наличия у пациента диабета, подобное высказывание о противодиабетических препаратах будет неверным. Известно, что некоторые препараты для лечения диабета повышают риск госпитализаций в связи с СН (росиглитазон, пиоглитазон, саксаглиптин), и поэтому они противопоказаны пациентам с СД2 и СН или с риском ее развития. Однако, несмотря на большое количество опубликованных исследований, по-прежнему существует неопределенность в отношении безопасности ряда противодиабетических препаратов у больных СН. В обзоре рассматриваются подходы к лечению СД у больных СН.

КЛЮЧЕВЫЕ СЛОВА: сахарный диабет; сердечная недостаточность; диабетическая кардиомиопатия; коморбидность

\title{
HEART FAILURE AND DIABETES MELLITUS: INSIGHT INTO COMORBIDITY
}

(c) Nina A. Petunina, Ivan V. Trukhin, Liubov V. Trukhina, Zhanna M. Sizova, Valeria L. Zakharova

I.M. Sechenov First Moscow State Medical University (Sechenov University), Moscow, Russia

Diabetes mellitus (DM) and heart failure (HF) are frequent comorbidities with a bidirectional relationship. Patients with HF have increased risk of developing DM, and those with DM are at greater risk of developing HF. HF does not fit clearly into the microangiopathy and macroangiopathy groups. It is known that coronary artery disease and arterial hypertension are the major causes of HF; however, it has been shown that DM can trigger functional and structural abnormalities in the myocardium via diabetic cardiomyopathy, a condition with either restrictive or dilated phenotype. While HF treatment is equally effective and safe in patients with and without DM, this statement is not applicable for antidiabetic treatment. Several antidiabetic drugs, such as rosiglitazone, pioglitazone and saxagliptin increase the risk of hospitalisation for HF, therefore these antidiabetic drugs are contraindicated in patients with DM and HF or patients at risk of developing HF. Despite a large number of clinical evidence, uncertainty about the safety of antidiabetic drugs in patients with HF always exists. In this review, the issues of DM treatment in patients with HF are addressed in detail.

\section{KEYWORDS: diabetes mellitus; heart failure; diabetic cardiomyopathy; comorbidity}

Сахарный диабет (СД) и сердечная недостаточность (CH) часто сосуществуют, что оказывает значительное влияние на выбор их лечения и прогноз. Пациенты с СД 2 типа (СД2) и СН как с сохранной, так и со сниженной фракцией выброса (ФВ) имеют более высокий функциональный класс $\mathrm{CH}$ по классификации NYHA по сравнению с больными без диабета даже при одинаковой ФВ [1]. Взаимосвязь между СД и СН двунаправленная: прогноз у пациентов с СН хуже, если у них есть СД, а пациенты с СД подвержены большему риску развития $\mathrm{CH}$. Не только наличие СД, но и качество контроля гликемии ассоциировано с риском развития СН. При увеличении гликированного гемоглобина $\left(\mathrm{HbA}_{1 c}\right)$ на $1 \%$ риск развития $\mathrm{CH}$ повышается на 8\% [2]. СН четко не вписывается как в группу микрососудистых, так и в группу макрососудистых осложнений СД. Несмотря на распространен- ность и высокую смертность, СН долгое время занимала едва ли не последнее место в иерархии осложнений СД. В своей статье Bell справедливо назвал $\mathrm{CH}$ «частым, забытым и зачастую фатальным осложнением СД» [3].

\section{ЭПИДЕМИОЛОГИЯ}

Хроническая СН и СД являются достаточно распространенными заболеваниями. По данным федерального регистра, 3\% населения Российской Федерации состоят на диспансерном учете с СД, а фактическая распространенность, по мнению экспертов, составляет около $6 \%$ населения [4]. В развитых странах распространенность $\mathrm{CH}$ составляет 1-2\% среди взрослого населения, достигая $10 \%$ и более у лиц старше 70 лет [5]. По другим данным, до 4,3\% взрослого населения в развитых странах стра- 
дают $\mathrm{CH}$, при этом $\mathrm{CH}$ с сохранной ФВ встречается чаще, чем СН со сниженной ФВ [6].

Распространенность СД у больных ХСН выше, чем у людей без нее. В Англии и Дании приблизительно 25\% пациентов с СН имеют СД2 [7]. В клинических исследованиях у больных СН распространенность СД2 составляла приблизительно 30\%, вне зависимости от фенотипа CH [8]. В исследовании Reykjavik распространенность ХСН у больных СД2 составила 12\% [9].

СД2 является независимым фактором риска развития СН. Еще в 1974 г. во Фремингемском исследовании было показано, что СД увеличивает риск развития СН в 2 раза у мужчин и в 5 раз у женщин [10]. В ретроспективном исследовании, проведенном Nichols и соавт., СН развивалась в 2,5 раза чаще у больных с СД, по сравнению с пациентами без диабета [11]. В проведенном недавно популяционном исследовании, включавшем 1,9 млн пациентов с СД2 без явных признаков сердечно-сосудистых заболеваний, сроком наблюдения 5,5 лет, заболеваемость СН (14,1\%) была выше, чем заболеваемость инфарктом миокарда (ИМ) или инсультом [12].

В наблюдательных исследованиях было показано, что больные СН имеют повышенный риск развития СД по сравнению с пациентами без СН. В исследовании, охватившем 20\% населения Портланда, штат Орегон, США, заболеваемость СД была существенно выше у пациентов с СН, чем у людей без нее $(13,6 / 1000$ и 9,2/1000 соответственно) за пятилетний период наблюдения [13]. В исследовании EMPHASIS-HF (Eplerenone in Mild Patients Hospitalization And Survlval Study in Heart Failure), включавшем больных ХСН со сниженной ФВ, заболеваемость СД2 составила 3,7\% при медиане наблюдения 21 мес. Важно отметить, что лечение ингибиторами ангиотензинпревращающего фермента (иАПФ) привело к снижению заболеваемости СД2 у больных СН со сниженной ФВ. В субанализе исследования SOLVD (Studies of Left Ventricular Dysfunction) у 6\% пациентов, принимавших эналаприл, развился СД2 при медиане наблюдения 2,9 года, по сравнению с 22\% в группе плацебо.

Данные наблюдательных исследований указывают на значительное количество пациентов старше 60 лет с недиагностированной СН (22,9\% и 4,8\%, СН с сохранной и сниженной ФВ соответственно) у больных СД [14]. Верно и обратное: недиагностированный преддиабет и СД2 не редкость у больных с CH. В исследовании RESOLVD (Randomized Evaluation of Strategies for Left Ventricular Dysfunction Ongoing Trial) у 11\% пациентов без СД2 был затем выявлен диабет.

\section{ПАТОГЕНЕЗ}

Патогенез СН при СД включает «кардиотоксическую тетраду»: ишемическую болезнь сердца (ИБС), артериальную гипертензию (АГ), диабетическую кардиомиопатию и увеличение объема внеклеточной жидкости [3]. Чаще всего причинами развития СН у больных СД являются ИБС и АГ [8]. Атеросклероз коронарных артерий у больных СД развивается в более молодом возрасте и поражает дистальные сегменты коронарных артерий. Помимо диффузного поражения коронарных артерий, в ответ на ишемию у больных СД коллатеральное кровообращение развивается хуже, чем у больных без СД, что, возможно, связано с нарушением продукции или ответа на сосудистые факторы роста [15]. Риск развития $\mathrm{CH}$ после ИМ у больных СД повышен в 2-3 раза [16]. Также существует гипотеза о том, что СД может непосредственно влиять на структуру и функцию сердца [17]. В 1954 г. Lundbaek предположил, что СД может напрямую вызывать дисфункцию миокарда без наличия ИБС и АГ. Позднее он назвал это состояние диабетической кардиомиопатией [18]. Почти на 20 лет позже Rubler и coавт. предоставили новые данные, свидетельствующие о том, что дисфункция миокарда может напрямую быть следствием СД, а не просто развиваться как следствие ИБС [19]. Диабетическая кардиомиопатия впервые была описана как состояние с дилатационным фенотипом с эксцентричным ремоделированием левого желудочка (ЛЖ) и систолической дисфункцией. Однако в последнее время в клинических исследованиях диабетическая кардиомиопатия описывается как состояние с рестриктивным фенотипом, концентрическим ремоделированием Лж и его диастолической дисфункцией, что послужило поводом выделить два ее фенотипа и назвать «двуликой болезнью» [17]. К сожалению, общепризнанного определения диабетической кардиомиопатии не существует, что затрудняет исследование ее эпидемиологии, патофизиологии, клинических проявлений и исходов [8]. Это состояние можно клинически определить как нарушение структуры или функции миокарда без поражения эпикардиальных коронарных артерий, гипертензии и значимых клапанных пороков. Однако по-прежнему продолжается дискуссия о существовании диабетической кардиомиопатии как самостоятельной нозологии. Хотя диабетическая кардиомиопатия не является обязательным условием для развития $\mathrm{CH}$, она повышает риск ее развития [20]. Существуют структурные и функциональные отличия между двумя фенотипами диабетической кардиомиопатии. При рестриктивном фенотипе наблюдается гипертрофия кардиомиоцитов с сохранением нормальной структуры саркомеров и реактивный фиброз, в то время как при дилатационном фенотипе повреждение кардиомиоцитов с утратой саркомеров, количество межклеточного коллагена при этом высокое и присутствует заместительный фиброз. При обоих фенотипах наблюдаются редукция микроциркуляторного русла и отложение в нем конечных продуктов гликирования [17].

Было идентифицировано множество механизмов, вносящих свой вклад в развитие диабетической кардиомиопатии, и между каждым из них существует тесная взаимосвязь. Гипергликемия, нарушение метаболизма свободных жирных кислот и липотоксичность, ускорение апоптоза, автономная нейропатия, микрососудистое поражение и эндотелиальная дисфункция, инсулинорезистентность и гиперинсулинемия, - лишь некоторые из описанных механизмов. Однако их вклад в развитие двух фенотипов СН различен. При рестриктивном фенотипе диабетической кардиомиопатии микроваскулярная эндотелиальная дисфункция приводит к ремоделированию Лж из-за снижения биодоступности оксида азота (NO) и сниженной активности протеинкиназы C [17]. Гипертрофия миокарда развивается в результате как отложения триглицеридов, так и увеличения объема межклеточного пространства за счет отложения коллагена. 
В миокарде обнаруживается периваскулярный фиброз при отсутствии ИБС и АГ, что может быть связано с повышением экспрессии трансформирующего фактора роста бета (TGF- $\beta$ ) и фактора роста соединительной ткани (CTGF) и одновременно с пониженным разрушением коллагена в связи со снижением экспрессии матриксных металлопротеаз, в частности матриксной металлопротеазы-2 (MМР-2). Конечные продукты гликирования вызывают образование сшивок между молекулами коллагена, что затрудняет его деградацию, ведет к повышению ригидности миокарда, нарушая его диастолическое расслабление [21]. Кроме того, гиперинсулинемия как следствие инсулинорезистентности может напрямую способствовать развитию гипертрофии миокарда [22]. При дилатационном фенотипе к ремоделированию и дисфункции ЛЖ приводит гибель кардиомиоцитов. Оксидативный стресс, тканевая гипоксия из-за разрежения микроциркуляторного русла, инфильтрация провоспалительными иммунокомпетентными клетками, конечные продукты гликирования и липотоксичность ведут к гибели кардиомиоцитов, что сопровождается заместительным фиброзом, которому способствует высокая активность протеинкиназы С в фибробластах [17].

Гипергликемия ведет к делению митохондрий в эндотелиоцитах и образованию супероксиданиона, что ассоциируется с нарушением функции NO-синтазы и снижением образования циклического гуанозинмонофосфата. Это приводит к снижению активности протеинкиназы С в прилегающих кардиомиоцитах и снижает их растяжимость, что вносит вклад в развитие диастолической ригидности миокарда ЛЖ, которая наблюдается при рестриктивном фенотипе СН [23]. Кроме того, гипергликемия вызывает активацию протеинкиназы С в фибробластах, что приводит к увеличению продукции и отложению коллагена [24].

Кардиальная автономная нейропатия представляет собой поражение вегетативных нервных волокон, иннервирующих сердце и кровеносные сосуды, в результате чего нарушается контроль частоты сердечных сокращений (ЧСС) и сосудистой гемодинамики. Клинические проявления кардиальной автономной нейропатии разнообразны и включают тахикардию, ортостатическую гипотензию, нарушение толерантности к физической нагрузке, периоперационную лабильность сердечно-сосудистой системы, безболевую форму ИМ. Возможно, кардиальная автономная нейропатия вносит вклад в развитие диастолической дисфункции ЛЖ. При кардиальной автономной нейропатии поражение блуждающего нерва приводит к относительному преобладанию симпатической активности, в результате чего активируется система ренин-ангиотензин-альдостерон (РААС), увеличиваются чСС, ударный объем и периферическое сосудистое сопротивление, что, в свою очередь, ведет к дисфункции миокарда Лж [25]. Гиперактивность симпатической нервной системы и локальная симпатическая денервация миокарда приводят к снижению резерва коронарного кровотока и диастолической дисфункции [26].

При возбуждении кардиомиоцитов происходит открытие потенциалзависимых кальциевых каналов клеточной мембраны и поступление ионов $\mathrm{Ca}^{2+}$ в цитоплазму, что, в свою очередь, вызывает выход ионов $\mathrm{Ca}^{2+}$ из саркоплазматического ретикулума. В результате этого происходит взаимодействие актина и миозина и сокращение кардиомиоцита. Расслабление наступает, когда ионы $\mathrm{Ca}^{2+}$ активно транспортируются обратно в саркоплазматический ретикулум с помощью $\mathrm{Ca}^{2+}$-АТФазы саркоплазматического ретикулума (SERCA-2). При инкубации кардиомиоцитов в растворе с высоким содержанием глюкозы было показано, что из-за оксидативного стресса активность SERCA-2 снижается, что ведет к перегрузке цитозоля кальцием, и, как следствие этого, нарушению релаксации. Нарушение гомеостаза $\mathrm{Ca}^{2+}$ ассоциируется с ремоделированием миофибрилл, а клинически - с диастолической дисфункцией миокарда [21].

У здоровых людей приблизительно одинаковое количество энергии, необходимой для сокращения кардиомиоцитов, выделяется при метаболизме глюкозы и свободных жирных кислот [20]. При СД утилизация глюкозы миокардом значительно снижается и происходит сдвиг в пользу бета-окисления жирных кислот. Причиной этого является снижение активности белков-транспортеров глюкозы ГЛЮТ-1 и ГЛЮТ-4. Повышенное поступление свободных жирных кислот в кардиомиоциты у больных $\mathrm{CH}$ превосходит возможность их окисления. Это ведет к накоплению триглицеридов в миокарде и в конечном итоге приводит к гибели клеток [27]. Свободные жирные кислоты ингибируют пируватдегидрогеназу, что нарушает энергетический обмен в миокарде и ведет к накоплению промежуточных продуктов гликолиза и церамида, приводящему к усилению апоптоза. Повышение захвата свободных жирных кислот миокардом было показано при проведении позитронно-эмиссионной томографии больным СД. У данных пациентов изменение утилизации энергетических субстратов ассоциировалось с повышенным потреблением кислорода миокардом и диастолической дисфункцией ЛЖ [28].

Как известно, СД является провоспалительным состоянием, а в многочисленных работах на мышах было показано повышение тканевого уровня цитокинов. В этих исследованиях было показано повышение количества межклеточных молекул адгезии ICAM-1, сосудистых молекул адгезии VCAM-1, инфильтрация ткани макрофагами и лейкоцитами, повышение экспрессии провоспалительных цитокинов: ИЛ-1, ИЛ-6, ИЛ-18, ФНО- $\alpha$, TGF- $\beta 1$. K сожалению, данные, подтверждающие наличие миокардиального воспаления у людей, отсутствуют [21].

Микрососудистые осложнения являются типичными для СД. Они включают нарушение проницаемости капилляров, формирование микроаневризм, утолщение базальной мембраны, фиброзирование стенок артериол. Резерв коронарного кровотока у больных СД снижен даже без обструктивного атеросклероза коронарных артерий и гипертрофии левого желудочка. Гипергликемия ведет к усиленному синтезу вазоконстрикторных простагландинов эндотелием, активации протеинкиназы C [20].

Многие механизмы, приводящие к развитию воспаления и фиброза в миокарде, могут активировать белки-регуляторы апоптоза или приводить непосредственно к некрозу, что наблюдается как при первом, так и втором типе СД. Эти механизмы включают повышенное образование активных форм кислорода, увеличение количества циркулирующих провоспалительных цитокинов и хемокинов, активацию каспаз, апоптоз, зависимый от активации Fas-рецептора (первый сигналь- 
ный рецептор апоптоза, CD-95), развитие резистентности к инсулиноподобному фактору роста 1 (ИФР-1), активацию тканевой РААС [21].

\section{ЛЕЧЕНИЕ}

Лечение $\mathrm{CH}$ у больных СД не имеет особых ограничений и должно проводиться в соответствии с рекомендациями Европейского общества кардиологов 2016 г. [5]. В клинических исследованиях было показано, что все виды лечения СН были одинаково эффективны вне зависимости от наличия у пациента СД. Тем не менее некоторые препараты, используемые в лечении $\mathrm{CH}$, проявляют метаболический эффект, который следует учитывать при лечении пациентов с СД.

Ингибиторы ангиотензин-превращающего фермента (ИАПФ) рекомендуются при отсутствии противопоказаний для всех пациентов с СН со сниженной ФВ, так как они показали снижение смертности и заболеваемости [5]. ИАПФ необходимо титровать до максимально переносимой дозы для достижения адекватного торможения РААС. Есть данные, свидетельствующие о том, что в клинической практике большинство пациентов получают субоптимальные дозы иАПФ [29]. Блокаторы рецепторов к ангиотензину (БРА) рекомендуется использовать только в качестве альтернативы у пациентов с непереносимостью иАПФ [30]. При СД существует высокий риск развития нефропатии и хронической болезни почек (ХБП), что ассоциируется с повышенной задержкой натрия и риском гиперкалиемии. Это следует учитывать при назначении иАПФ и БРА, поскольку эти препараты снижают экскрецию калия. Таким образом, при назначении иАПФ и БРА, а также при увеличении их дозировок следует проводить исследование электролитов плазмы крови.

Бета-блокаторы (ББ) обладают такой же эффективностью в отношении улучшения прогноза пациентов с СД, как и у пациентов без СД, при этом различные ББ могут оказывать неодинаковый эффект на показатели гликемии. В исследовании GEMINI было проведено прямое сравнение метопролола и карведилола по влиянию на гликемический контроль у больных СД. Было показано, что на фоне блокады РААС применение карведилола не повлияло на контроль гликемии и даже привело к улучшению нескольких компонентов метаболического синдрома, а также к замедлению развития альбуминурии. Применение метопролола ассоциировалось с незначительным повышением $\mathrm{HbA}_{1 с}$ [31]. Сравнение метопролола и карведилола также проводилось в исследовании СОMЕТ. Прием карведилола ассоциировался с меньшей заболеваемостью СД у пациентов с CH по сравнению с метопрололом [32]. Гипогликемия может быть еще большей проблемой у пациентов с СД2, которые получают инсулин или препараты сульфонилмочевины. Теоретически ББ могут маскировать адренергические проявления гипогликемии, препятствуя развитию тахикардии и тремора, а также увеличить время выхода из гипогликемического состояния, блокируя $\beta 2$-адренорецепторы, которые частично контролируют образование глюкозы в печени. Однако в исследовании MERIT-HF (Effect of metoprolol CR/XL in chronic heart failure: Metoprolol CR/XL Randomised Intervention Trial in Con- gestive Heart Failure) среди пациентов с СД всего лишь у 0,8\% в группе метопролола наблюдались нежелательные явления, связанные с гипогликемией, что сравнимо с 0,6\% в группе плацебо [33]. На основании результатов рандомизированных клинических исследований для лечения $\mathrm{CH}$ у больных СД2 рекомендованы следующие ББ: метопролола сукцинат (MERIT-HF), бисопролол (CIBIS II, The Cardiac Insufficiency Bisoprolol Study II), карведилол (COPERNICUS, The Carvedilol Prospective Randomized Cumulative Survival Trial, COMET, Comparison of carvedilol and metoprolol on clinical outcomes in patients with chronic heart failure in the Carvedilol Or Metoprolol European Trial) [8]. Подводя итоги, следует подчеркнуть, что польза от применения ББ у больных СД значительно перевешивает риски, связанные с гипогликемией, незначительным повышением уровней $\mathrm{HbA}_{1 с}$ и липидов плазмы.

Антагонисты альдостерона спиронолактон и эплеренон показали снижение смертности у пациентов как с СД, так и без СД с СН со сниженной ФВ, однако их совместное применение с иАПФ/БРА ограничено из-за опасений, связанных с ухудшением функции почек и гиперкалиемией [34]. Следует соблюдать осторожность при использовании антагонистов рецепторов минералокортикоидов у пациентов с нарушением функции почек и у пациентов с уровнем калия в сыворотке крови $>5$ ммоль/л [8].

В клинические рекомендации Европейского кардиологического общества вошел препарат из нового класса ингибиторов рецепторов ангиотензина-неприлизина (ARNI), действующий на PAAC и систему нейтральных эндопептидаз - сакубитрил/валсартан. В исследовании PARADIGM-HF (Angiotensin-neprilysin inhibition versus enalapril in heart failure) препарат сакубитрил/валсартан превзошел иАПФ эналаприл в снижении смертности и числа госпитализаций у больных с СН со сниженной ФВ, вне зависимости от наличия у них диабета. В проведенном post-hoc-анализе лечение препаратом сакубитрил/ валсартан ассоциировалось с большим снижением $\mathrm{HbA}_{1 c}$ и меньшей частотой начала инсулинотерапии по сравнению с эналаприлом [35].

Диуретики обычно используются для уменьшения симптомов задержки жидкости у пациентов с СН. Рандомизированных клинических исследований, оценивающих их эффективность у пациентов с СН и СД, на данный момент нет. Однако в кохрейновском систематическом обзоре, включившем 14 исследований (525 больных), было показано, что у пациентов, принимающих петлевые и тиазидные диуретики, риск смерти и ухудшения течения СН был ниже, чем в группе плацебо. Теоретически тиазидные диуретики могут приводить к усилению инсулинорезистентности и последующему ухудшению контроля гликемии [8].

До сих пор нет доказанного лечения пациентов с CH с сохраненной ФВ и СН со средней ФВ, которое снижало бы заболеваемость и смертность этих пациентов, поэтому важной целью лечения является уменьшение симптомов $\mathrm{CH}$. Нет доказательств того, что ББ и антагонисты альдостерона уменьшают выраженность симптомов $\mathrm{CH}$ у данной группы больных, а данные относительно иАПФ и БРА противоречивы. Однако было показано, что диуретики уменьшают симптомы СН вне зависимости от ФВ ЛЖ [5].

Уже более 15 лет известно, что некоторые средства для лечения СД2 могут увеличивать риск СН. На данный 
момент показано, что две группы сахароснижающих препаратов - тиазолидиндионы и ингибиторы дипептидилпептидазы-4 (иДПП-4) (саксаглиптин), - могут увеличивать риск СН $[36,37]$. Показано, что сенситайзеры инсулина росиглитазон и пиоглитазон имеют выраженный антиатеросклеротический эффект, но, к сожалению, вызывают задержку натрия и жидкости посредством uр-регуляции аквапорина-1 в проксимальных отделах собирательных трубок, что и приводит к увеличению риска развития СH [38]. Однако в исследовании PROACTIV, включавшем больных с макрососудистыми осложнениями СД (критерии включения: перенесенный ИМ, чрескожное вмешательство или аорто-коронарное шунтирование, ИБС или облитерирующий атеросклероз артерий нижних конечностей), СН была диагностирована у $11 \%$ пациентов в группе пиоглитазона и $8 \%$ в группе плацебо ( $p<0,0001)$, только 6\% больных в группе плацебо и 4\% в группе пиоглитазона были госпитализированы в связи с СН $(\mathrm{p}=0,007)$, а смертность в связи с СН не различалась между группами ( $\mathrm{p}=0,634)$. Следует отметить, что в этом исследовании СН не была включена ни в первичную, ни во вторичную конечную точку [39]. В недавно опубликованном исследовании TOSCA.IT (Effects on the incidence of cardiovascular events of the addition of pioglitazone versus sulfonylureas in patients with type 2 diabetes inadequately controlled with metformin: a randomised, multicentre trial) проводилось сравнение пиоглитазона с препаратами сульфонилмочевины у больных, получавших метформин. В этом исследовании СН не была включена ни в первичную конечную точку, ни в группу основных вторичных конечных точек. Интересно, что в этом исследовании риск развития СН был одинаковым в обеих группах [40]. В исследовании SAVOR-TIMI 53 саксаглиптин значительно увеличивал риск госпитализаций по поводу CH [37]. На основании этих данных пиоглитазон, росиглитазон и саксаглиптин противопоказаны больным с $\mathrm{CH}$ или же риском ее развития. В то же время не все иДПП4 повышают риск СН. В исследовании EXAMINE применение алоглиптина не привело к статистически значимому увеличению риска госпитализации в связи с СН по сравнению с плацебо у больных, перенесших острый коронарный синдром [41]. Аналогично, в исследовании TECOS ситаглиптин не повлиял на риск госпитализаций с связи с СН у больных СД2, в том числе у пациентов высокого риска [42].

Долгое время существует опасение, что инсулин, приводя к задержке натрия и жидкости и увеличению массы тела, может увеличивать риск развития С. В крупных наблюдательных исследованиях было продемонстрировано, что лечение препаратами инсулина ассоциировано с более высокой смертностью, чем терапия метформином. Похожие опасения существуют и в отношении препаратов сульфонилмочевины, которые, будучи секретагогами инсулина, также ассоциированы с более высокой смертностью по сравнению с метформином [43]. Увеличение риска развития СН при применении препаратов сульфонилмочевины может быть связано не только со значительно более высоким риском гипогликемии и повышением массы тела, но и их негативным влиянием на ишемическое прекондиционирование. Однако результаты этих исследований неубедительны, так как они не были рандомизированными. В единственном на данный момент рандомизированном клиническом исследовании ORIGIN лечение инсулином по сравнению с плацебо не привело к увеличению госпитализаций по поводу $\mathrm{CH}$ (следует отметить, что в исследовании участвовали пациенты с факторами риска сердечно-сосудистых заболеваний, но без СН) [44]. В 2017 г. были опубликованы результаты исследования DEVOTE, в котором производилось сравнение инсулина деглудек с инсулином гларгин. 85,2\% участвующих в нем пациентов имели сердечно-сосудистые заболевания, ХБП или же обе патологии. Было показано, что сердечно-сосудистые события в группе деглудека происходили не чаще, чем в группе гларгина, однако в первичную конечную точку CH включена не была [45]. В исследованиях UKPDS и ADVANCE, где в основном применялись препараты сульфонилмочевины, увеличения риска СН показано не было $[46,47]$. Примечательно, что, несмотря на то что инсулин и препараты сульфонилмочевины используются уже многие десятилетия, других рандомизированных плацебо-контролируемых исследований нет.

Уже многие годы метформин является важным компонентом в терапии СД2. Ранее полагали, что СН является противопоказанием для назначения метформина, что в дальнейшем было пересмотрено. На основании результатов крупных наблюдательных исследований считается, что метформин эффективен и безопасен у пациентов с СД и СН. Более того, в нескольких исследованиях было показано, что метформин может даже снижать риск развития СН и смерти у больных СД. Однако главным ограничением является тот факт, что эти исследования были ретроспективными наблюдательными. Конечно, рандомизированное клиническое исследование (РКИ) по сравнению метформина с плацебо предоставило бы более надежные данные, но проведение подобного исследования фактически невозможно [48]. Согласно рекомендациям Европейского общества кардиологов, применение метформина безопасно у больных $\mathrm{CH}$ со сниженной ФВ, и, более того, он является препаратом выбора у больных СН [5]. В исследовании случай-контроль, включавшем больных с СН и СД, MacDonald и coавт. показали, что, по сравнению с пациентами, не получавшими антидиабетических препаратов, применение метформина ассоциировалось с более низким риском смерти, чем использование других сахароснижающих средств, даже с поправкой на уровень гликемического контроля, функцию почек, индекс массы тела и артериальное давление. В то же время использование других сахароснижающих средств или инсулина не привело к увеличению смертности от всех причин. На основании полученных данных исследователи сделали вывод, что очевидное преимущество метформина по сравнению с другими препаратами связано со снижением риска смерти при приеме метформина, нежели вреда при приеме других сахароснижающих средств [49]. Однако Rémy Boussageon и соавт. в опубликованном в 2012 г. мета-анализе показали, что лечение метформином не оказывает статистически значимого влияния на смертность от всех причин, сердечно-сосудистую смертность и заболеваемость сердечно-сосудистыми заболеваниями у пациентов с СД2. По данными Rémy Boussageon и соавт., нельзя исключить снижение на 33\% или же повышение на 64\% сердечно-сосудистой смертности [50]. В исследовании 
UKPDS у 34 пациентов с избытком массы тела (более 120\% идеальной массы тела), принимавших метформин, было показано снижение смертности по сравнению с контрольной группой. При сравнении метформина и препаратов сульфонилмочевины, а также метформина и диеты заболеваемость СН статистически не различалась. В то же время добавление метформина к препарату сульфонилмочевины ассоциировалось с увеличением риска связанной с диабетом смерти на $96 \%(p=0,039)$ и смерти от всех причин на 60\% $(p=0,041)$ [51]. Однако Josie M.M. Evans и соавт. показали, что у пациентов с СД и СН при применении метформина в качестве монотерапии или в комбинации с препаратом сульфонилмочевины наблюдалась меньшая смертность, чем при монотерапии препаратами сульфонилмочевины (исследовалась популяция региона Tayside, Шотландия) [52], что было подтверждено в исследовании, проведенном C. Andersson и соавт. в Дании [53].

Агонисты рецепторов глюкагоноподобного пептида-1 (аГПП-1) исследовались во многих плацебо-контролируемых исследованиях у больных СД2 и сердечно-сосудистыми заболеваниями или же с высоким риском их развития. В этих исследованиях аГПП-1 не влияли на число госпитализаций по поводу СН. В исследовании LIVE (Effect of liraglutide, a glucagon-like peptide-1 analogue, on left ventricular function in stable chronic heart failure patients with and without diabetes) оценивался эффект аГПП-1 лираглутида на функцию Лж у больных с СН (критерием включения была ФВ ЛЖ <45\%) с и без СД2. По сравнению с плацебо лираглутид не повлиял на систолическую функцию ЛЖ, однако применение лираглутида ассоциировалось с увеличением ЧСС ( $<<0,0001)$ и неблагоприятных явлений со стороны сердечно-сосудистой системы ( $\leq \leq 0,05)$, что требует дополнительных исследований по безопасности этого препарата в разных подгруппах больных с CH [54]. В исследовании LEADER количество госпитализаций в связи с СН в группе лираглутида было ниже, чем в группе плацебо, однако статистически эта разница была незначимой (4,7\% и 5,3\% соответственно; ДИ 0,87 $(0,73-1,05) ; P=0,14)[55]$. В проведенном post-hoc-анализе исследования LEADER в подгруппах с CH 2 и 3 функционального класса (ФК) по NYHA и без СН на момент начала исследования сравнивалась частота госпитализаций по поводу СН. В обеих подгруппах лечение лираглутидом не оказывало статистически значимого влияния на число госпитализаций по поводу СН [56].

Важным открытием последних лет в эндокринологии и кардиологии стало внедрение в клиническую практику новой группы сахароснижающих препаратов - ингибиторов натрий-глюкозного котранспортера 2 типа (иНГЛТ-2), назначение которых приводит к снижению числа госпитализаций по поводу СН у пациентов с сердечно-сосудистыми заболеваниями или высоким риском их развития. В двух больших РКИ, в которых оценивалась сердечно-сосудистая безопасность иНГЛТ-2, эмпаглифлозина и канаглифлозина, при применении обоих препаратов было показано значительное снижение количества госпитализаций по поводу $\mathrm{CH}[57,58]$.

Традиционно для достижения целевых значений гликемии у больных СД2 терапевтические вмешательства были направлены на восстановление активности $\beta$-клеток, чувствительности к инсулину и тканевого захвата глюкозы. ИНГЛТ-2 имеют новый механизм действия: снижают реабсорбцию глюкозы в почках. Уже в исследованиях 1930-х гг. была показана важная роль почек в гомеостазе глюкозы. Было продемонстрировано, что почти вся профильтрованная в клубочках глюкоза подвергается канальцевой реабсорбции, за исключением случаев, когда порог реабсорбции превышен, а при этом глюкозурия линейно повышается с увеличением глюкозы плазмы. Пятьюдесятью годами позднее были описаны 2 транспортные системы: НГЛТ-1 и НГЛТ-2. НГЛТ-1 был обнаружен в клетках, изолированных из кишечника, сердца, скелетной мускулатуры и почек. НГЛТ-2 располагается фактически только в эпителии проксимальных извитых канальцев и отвечает за реабсорбцию практически всей профильтрованной глюкозы. В исследованиях на мышах было показано, что с помощью НГЛТ-2 реабсорбируется 97\% глюкозы, в то время как оставшиеся 3\% реабсорбируются с помощью НГЛТ-1 в более дистальных отделах нефрона. Также было показано, что экспрессия НГлТ-2 повышается при гипергликемии, что, скорее всего, отражает адаптивный ответ на увеличенную фильтрацию глюкозы, а также парадоксально увеличивает порог экскреции глюкозы с мочой у больных СД [59].

В настоящее время зарегистрировано 3 препарата из группы иНГЛТ-2: эмпаглифлозин, канаглифлозин и дапаглифлозин. ИНГЛТ-2 препятствуют реабсорбции натрия и глюкозы в проксимальных извитых канальцах, что приводит к глюкозурии и натрийурии. Действие иНГЛТ-2 не зависит от уровня инсулина, функции поджелудочной железы и степени инсулинорезистентности. Другой отличительной чертой этих препаратов является низкий риск гипогликемии. Этот класс лекарственных средств приводит к снижению АД, микроальбуминурии (маркер сердечно-сосудистого риска), оказывает урикозурическое действие, снижает жесткость артерий и повышает холестерин липопротеинов высокой и низкой плотности (ЛПНП и ЛПВП), не изменяя соотношение ЛПНП/ЛПВП[60].

Хотя механизмы, ответственные за снижение АД при применении иНГЛТ-2, до конца не изучены, считается, что наиболее важными из них являются изменение объема плазмы и снижение артериальной жесткости. Было показано, что за 12-недельный период наблюдения, по сравнению с плацебо или приемом тиазидных диуретиков, терапия дапаглифлозином привела к снижению объема плазмы на 7\% и увеличению гематокрита [61]. Увеличенная артериальная жесткость ассоциирована с гипертензией, гипергликемией, ожирением, эхокардиографическими признаками СН. Более того, увеличенная артериальная жесткость является предиктором сердечно-сосудистых катастроф, СН и смерти, особенно у пациентов с СД и высоким сердечно-сосудистым риском. У больных СД на фоне приема иНГЛТ-2 наблюдалось снижение пульсового давления и амбулаторного индекса артериальной жесткости (маркеры артериальной жесткости) [62].

В исследовании EMPA-REG OUTCOME, включавшем больных с СД2 и сердечно-сосудистой патологией, было показано значительное снижение госпитализаций по поводу СН (отношение шансов 0,65) у пациентов, принимавших эмпаглифлозин по сравнению с плацебо. Этот благоприятный эффект эмпаглифлозина наблюдался уже 
через 2-3 месяца от начала лечения, причем как у больных, имевших СН на момент начала исследования, так и у больных без нее. Несмотря на высокую смертность госпитализированных пациентов, у принимавших эмпаглифлозин она была ниже, чем в группе плацебо (13,5\% и 24,2\% соответственно). Кроме того, по сравнению с плацебо, прием эмпаглифлозина ассоциировался со снижением массы тела, окружности талии, уровня мочевой кислоты, систолического и диастолического давления (без увеличения чСС) и небольшим увеличением холестерина ЛПВП и ЛПНП [57].

В исследовании CANVAS, включавшем пациентов с СД2 и сердечно-сосудистой патологией (65\% больных) или с высоким риском ее развития, было показано значительное снижение госпитализаций по поводу CH (отношение рисков 0,67; ДИ 0,52-0,87) у пациентов, принимавших канаглифлозин по сравнению с группой плацебо [58]. СН имели 14\% больных, вошедших в это исследование. Эмпаглифлозин в исследовании EMPA-REG OUTCOME, но не канаглифлозин в исследовании CANVAS привел не только к снижению числа госпитализаций по поводу $\mathrm{CH}$, но и к снижению смертности от всех причин и сердечно-сосудистой смертности [57, 58]. B post-hoc-анализе исследования EMPA-REG OUTCOME было проведено сравнение числа госпитализаций по поводу $\mathrm{CH}$ в подгруппах пациентов с и без $\mathrm{CH}$ на момент начала исследования. В подгруппе пациентов без СH на момент начала исследования 1,8\% были госпитализированы в связи с СН в группе эмпаглифлозина и 3,2\% в группе плацебо (отношение рисков 0,59, ДИ 0,43-0,82). В подгруппе пациентов с СН на момент начала исследования 10,4\% были госпитализированы в связи с СH в группе эмпаглифлозина и 12,3\% в группе плацебо (отношение рисков 0,75; ДИ 0,48-1,19) [63].

В конце 2018 г. будут опубликованы результаты многоцентрового исследования DECLARE-TIMI 58, включившего более 17 тысяч больных СД с несколькими факторами риска сердечно-сосудистых заболеваний или с установленной сердечно-сосудистой патологией. В исследовании оценивали влияние терапии дапаглифлозином как на комбинированную конечную точку (серьезные неблагоприятные сердечно-сосудистые события), так и на первичную конечную точку, включающую госпитализацию по поводу СН и/или сердечно-сосудистую смерть.

Еще ни один из препаратов для лечения СД2 не исследовался как средство лечения СН. В 2017 г. стартовали 3 больших РКИ с иНГЛТ, которые будут включать пациентов с СН как с СД, так и без диабета. В двух исследованиях (EMPEROR-Reduced, Empagliflozin outcome trial in patients with chronic heart failure with reduced ejection fraction, и EMPEROR-Preserved, Empagliflozin outcome trial in patients with chronic heart failure with preserved ejection fraction) будет проведена оценка эффективности и безопасности эмпаглифлозина по сравнению с плацебо у пациентов, получающих терапию в соответствии с клиническими рекомендациями по лечению $\mathrm{CH}$ со сниженной и сохранной ФВ соответственно. В третьем исследовании (Dapa-HF, Study to Evaluate the Effect of Dapagliflozin on the Incidence of Worsening Heart Failure or Cardiovascular Death in Patients With Chronic Heart Failure) будет оценена эффективность и безопасность дапаглифлозина по сравнению с плацебо по влиянию на сердечно-сосудистую смертность и госпитализации в связи с $\mathrm{CH}$ у пациентов с СН со сниженной ФВ.

\section{выводы}

СД2 и СН являются довольно распространенными заболеваниями и часто сосуществуют. В то время как основными причинами развития СН у больных СД2 являются ИБС и АГ, развитие диабетической кардиомиопатии также вносит свой вклад. Данные из последних крупных клинических исследований и регистров свидетельствуют о значительно повышенном риске госпитализаций и смерти у больных СД2 и СН, вне зависимости от ее этиологии и фенотипа. Все медикаментозные и немедикаментозные виды лечения СН одинаково эффективны вне зависимости от наличия у пациента диабета. По-прежнему существует неопределенность в отношении безопасности препаратов сульфонилмочевины и инсулина у больных СД2 и СН, в то время как некоторые препараты для лечения диабета повышают риск госпитализаций в связи с СН (росиглитазон, пиоглитазон, саксаглиптин), и поэтому они противопоказаны пациентам с СД2 и СН или с риском ее развития. Кроме того, было показано, что иНГЛТ-2 эмпаглифлозин и канаглифлозин снижают риск госпитализаций в связи с СН у больных СД2. В настоящее время проводятся исследования иНГЛТ-2 как потенциального компонента терапии $\mathrm{CH}$ как у пациентов с СД2, так и у больных без диабета.

\section{ДОПОЛНИТЕЛЬНАЯ ИНФОРМАЦИЯ}

Конфликт интересов. Авторы декларируют отсутствие явных и потенциальных конфликтов интересов, связанных с публикацией настоящей статьи.

Участие авторов. Петунина Н.А. - концепция и дизайн статьи, анализ данных, редактирование; Трухин В.И. - сбор материала, анализ данных, подготовка текста статьи; Трухина Л.В. - анализ данных, редактирование; Сизова Ж.М. - концепция и дизайн статьи, анализ данных, редактирование; Захарова В.Л. - анализ данных, редактирование. Все авторы внесли существенный вклад в проведение анализа исследований, подготовку статьи, прочли и одобрили финальную версию перед публикацией.

\section{СПИСОК ЛИТЕРАТУРЫ| REFERENCES}

1. Suskin N, McKelvie RS, Burns RJ, et al. Glucose and insulin abnormalities relate to functional capacity in patients with congestive heart failure. Eur Heart J. 2000;21(16):1368-1375. doi: 10.1053/ euhj.1999.2043

2. McMurray JJV, Gerstein HC, Holman RR, Pfeffer MA. Heart failure: a cardiovascular outcome in diabetes that can no longer be ignored. Lancet Diabetes Endocrinol. 2014;2(10):843-851. doi: 10.1016/s22138587(14)70031-2
3. Bell DSH. Heart Failure: The frequent, forgotten, and often fatal complication of diabetes. Diabetes Care. 2003;26(8):2433-2441. doi: 10.2337/diacare.26.8.2433

4. Дедов И.И., Шестакова М.В., Майоров А.Ю., и др. Алгоритмы специализированной медицинской помощи больным сахарным диабетом. / Под ред. Дедова И.И., Шестаковой М.В., Майорова А.Ю. - 8-й выпуск // Сахарный диабет. - 2017. - Т. 20. №1S. - C. 1-121. [Dedov II, Shestakova MV, Mayorov AY, et al. De- 
dov II, Shestakova MV, Mayorov AY, editors. Standards of specialized diabetes care. Edited by. 8th ed. Diabetes mellitus. 2017;20(1S):1-121. (In Russ.)] doi: 10.14341/DM2017158

5. Ponikowski P, Voors AA, Anker SD, et al. 2016 ESC Guidelines for the diagnosis and treatment of acute and chronic heart failure: The Task Force for the diagnosis and treatment of acute and chronic heart failure of the European Society of Cardiology (ESC)Developed with the special contribution of the Heart Failure Association (HFA) of the ESC. Eur Heart J. 2016;37(27):2129-2200. doi: 10.1093/eurheartj/ehw128

6. van Riet EE, Hoes AW, Wagenaar KP, et al. Epidemiology of heart failure: the prevalence of heart failure and ventricular dysfunction in older adults over time. A systematic review. Eur J Heart Fail. 2016;18(3):242-252. doi: 10.1002/ejhf.483

7. Davies MK, Hobbs FDR, Davis RC, et al. Prevalence of left-ventricular systolic dysfunction and heart failure in the Echocardiographic Heart of England Screening study: a population based study. The Lancet. 2001;358(9280):439-444. doi: 10.1016/s0140-6736(01)05620-3

8. Seferovic PM, Petrie MC, Filippatos GS, et al. Type 2 diabetes mellitus and heart failure: a position statement from the Heart Failure Association of the European Society of Cardiology. Eur J Heart Fail. 2018;20(5):853-872. doi: 10.1002/ejhf.1170

9. Thrainsdottir IS, Aspelund T, Thorgeirsson G, et al. The Association Between Glucose Abnormalities and Heart Failure in the Population-Based Reykjavik Study. Diabetes Care. 2005;28(3):612-616. doi: 10.2337/diacare.28.3.612

10. Kannel WB, Hjortland M, Castelli WP. Role of diabetes in congestive heart failure: The Framingham study. The Am J Cardiol. 1974;34(1):29-34. doi: 10.1016/0002-9149(74)90089-7

11. Nichols GA, Gullion CM, Koro CE, et al. The Incidence of Congestive Heart Failure in Type 2 Diabetes: An update. Diabetes Care. 2004;27(8):1879-1884. doi: 10.2337/diacare.27.8.1879

12. Shah AD, Langenberg C, Rapsomaniki E, et al. Type 2 diabetes and incidence of cardiovascular diseases: a cohort study in 1.9 million people. Lancet Diabetes Endocrinol. 2015;3(2):105-113. doi: 10.1016/s2213-8587(14)70219-0

13. Nichols GA, Hillier TA, Erbey JR, Brown JB. Congestive Heart Failure in Type 2 Diabetes: Prevalence, incidence, and risk factors. Diabetes Care. 2001;24(9):1614-1619. doi: 10.2337/diacare.24.9.1614

14. Boonman-de Winter LJ, Rutten FH, Cramer MJ, et al. High prevalence of previously unknown heart failure and left ventricular dysfunction in patients with type 2 diabetes. Diabetologia. 2012;55(8):2154-2162. doi: 10.1007/s00125-012-2579-0

15. Chou E, Suzuma I, Way KJ, et al. Decreased Cardiac Expression of Vascular Endothelial Growth Factor and Its Receptors in Insulin-Resistant and Diabetic States. Circulation. 2002;105(3):373-379. doi: 10.1161/hc0302.102143

16. Stone PH, Muller JE, Hartwell T, et al. The effect of diabetes mellitus on prognosis and serial left ventricular function after acute myocardial infarction: Contribution of both coronary disease and diastolic left ventricular dysfunction to the adverse prognosis. J Am Coll Cardiol. 1989;14(1):49-57. doi: 10.1016/0735-1097(89)90053-3

17. Seferovic PM, Paulus WJ. Clinical diabetic cardiomyopathy: a twofaced disease with restrictive and dilated phenotypes. Eur Heart J. 2015;36(27):1718-1727, 1727a-1727c. doi: 10.1093/eurheartj/ehv134

18. Lundbæk K. Diabetic Angiopathy. Lancet. 1954;263(6808):377-379. doi: 10.1016/s0140-6736(54)90924-1

19. Rubler S, Dlugash J, Yuceoglu YZ, et al. New type of cardiomyopathy associated with diabetic glomerulosclerosis. Am J Cardiol. 1972;30(6):595-602. doi: 10.1016/0002-9149(72)90595-4

20. Aneja A, Tang WH, Bansilal S, et al. Diabetic cardiomyopathy: insights into pathogenesis, diagnostic challenges, and therapeutic options. Am J Med. 2008;121(9):748-757. doi: 10.1016/j.amjmed.2008.03.046

21. Bugger $\mathrm{H}$, Abel ED. Molecular mechanisms of diabetic cardiomyopathy. Diabetologia. 2014;57(4):660-671. doi: 10.1007/s00125-014-3171-6

22. Shimizu I, Minamino T, Toko H, et al. Excessive cardiac insulin signaling exacerbates systolic dysfunction induced by pressure overload in rodents. J Clin Invest. 2010;120(5):1506-1514. doi: 10.1172/JCI40096

23. Hamdani N, Bishu KG, von Frieling-Salewsky M, et al. Deranged myofilament phosphorylation and function in experimental heart failure with preserved ejection fraction. Cardiovasc Res. 2013;97(3):464-471. doi: 10.1093/cvr/cvs353

24. Asbun J, Villarreal FJ. The pathogenesis of myocardial fibrosis in the setting of diabetic cardiomyopathy. J Am Coll Cardiol. 2006;47(4):693-700. doi: 10.1016/j.jacc.2005.09.050
25. Perin PC, Maule S, Quadri R. Sympathetic Nervous System, Diabetes, and Hypertension. Clin Exp Hypertens. 2001;23(1-2):45-55. doi: 10.1081/ceh-100001196

26. Pop-Busui R, Kirkwood I, Schmid H, et al. Sympathetic dysfunction in type 1 diabetes: association with impaired myocardial blood flow reserve and diastolic dysfunction. J Am Coll Cardiol. 2004;44(12):2368-2374. doi: 10.1016/j.jacc.2004.09.033

27. Gonzalez A, Ravassa S, Beaumont J, et al. New targets to treat the structural remodeling of the myocardium. J Am Coll Cardiol. 2011;58(18):1833-1843. doi: 10.1016/j.jacc.2011.06.058

28. Rijzewijk LJ, van der Meer RW, Lamb HJ, et al. Altered myocardial substrate metabolism and decreased diastolic function in nonischemic human diabetic cardiomyopathy: studies with cardiac positron emission tomography and magnetic resonance imaging. J Am Coll Cardiol. 2009;54(16):1524-1532. doi: 10.1016/j.jacc.2009.04.074

29. Maggioni AP, Anker SD, Dahlstrom U, et al. Are hospitalized or ambulatory patients with heart failure treated in accordance with European Society of Cardiology guidelines? Evidence from 12,440 patients of the ESC Heart Failure Long-Term Registry. Eur J Heart Fail. 2013;15(10):1173-1184. doi: 10.1093/eurjhf/hft134

30. Granger CB, McMurray JJV, Yusuf S, et al. Effects of candesartan in patients with chronic heart failure and reduced left-ventricular systolic function intolerant to angiotensin-converting-enzyme inhibitors: the CHARM-Alternative trial. Lancet. 2003;362(9386):772-776. doi: 10.1016/s0140-6736(03)14284-5

31. Bakris GL, Fonseca V, Katholi RE, et al. Metabolic effects of carvedilol vs metoprolol in patients with type 2 diabetes mellitus and hypertension: a randomized controlled trial. JAMA. 2004;292(18):2227-2236. doi: 10.1001/jama.292.18.2227

32. Torp-Pedersen C, Metra M, Charlesworth A, et al. Effects of metoprolol and carvedilol on pre-existing and new onset diabetes in patients with chronic heart failure: data from the Carvedilol Or Metoprolol European Trial (COMET). Heart. 2007;93(8):968-973. doi: 10.1136/hrt.2006.092379

33. Deedwania PC, Giles TD, Klibaner M, et al. Efficacy, safety and tolerability of metoprolol CR/XL in patients with diabetes and chronic heart failure: experiences from MERIT-HF. Am Heart J. 2005;149(1):159-167. doi: 10.1016/j.ahj.2004.05.056

34. Dei Cas A, Khan SS, Butler J, et al. Impact of diabetes on epidemiology, treatment, and outcomes of patients with heart failure. JACC Heart Fail. 2015;3(2):136-145. doi: 10.1016/j.jchf.2014.08.004

35. Seferovic JP, Claggett B, Seidelmann SB, et al. Effect of sacubitril/ valsartan versus enalapril on glycaemic control in patients with heart failure and diabetes: a post-hoc analysis from the PARADIGM-HF trial. Lancet Diabetes Endocrinol. 2017;5(5):333-340. doi: 10.1016/s2213-8587(17)30087-6

36. Scirica BM, Braunwald E, Raz I, et al. Heart failure, saxagliptin, and diabetes mellitus: observations from the SAVOR-TIMI 53 randomized trial. Circulation. 2014;130(18):1579-1588. doi: 10.1161/CIRCULATIONAHA.114.010389

37. Lago RM, Singh PP, Nesto RW. Congestive heart failure and cardiovascular death in patients with prediabetes and type 2 diabetes given thiazolidinediones: a meta-analysis of randomised clinical trials. Lancet. 2007;370(9593):1129-1136. doi: 10.1016/s0140-6736(07)61514-1

38. Saad S, Zhang J, Yong R, et al. Role of the EGF receptor in PPARgamma-mediated sodium and water transport in human proximal tubule cells. Diabetologia. 2013;56(5):1174-1182. doi: 10.1007/s00125-013-2835-y

39. Dormandy JA, Charbonnel B, Eckland DJA, et al. Secondary prevention of macrovascular events in patients with type 2 diabetes in the PROactive Study (PROspective pioglitAzone Clinical Trial In macroVascular Events): a randomised controlled trial. Lancet. 2005:366(9493):1279-1289. doi: 10.1016/s0140-6736(05)67528-9

40. Vaccaro O, Masulli M, Nicolucci A, et al. Effects on the incidence of cardiovascular events of the addition of pioglitazone versus sulfonylureas in patients with type 2 diabetes inadequately controlled with metformin (TOSCA.IT): a randomised, multicentre trial. Lancet Diabetes Endocrinol. 2017;5(11):887-897. doi: 10.1016/s2213-8587(17)30317-0

41. White WB, Cannon CP, Heller SR, et al. Alogliptin after acute coronary syndrome in patients with type 2 diabetes. N Engl J Med. 2013;369(14):1327-1335. doi: 10.1056/NEJMoa1305889

42. McGuire DK, Van de Werf F, Armstrong PW, et al. Association Between Sitagliptin Use and Heart Failure Hospitalization and Related Outcomes in Type 2 Diabetes Mellitus: Secondary Analysis 
of a Randomized Clinical Trial. JAMA Cardiol. 2016;1 (2):126-135. doi: 10.1001/jamacardio.2016.0103

43. MacDonald MR, Petrie MC, Hawkins NM, et al. Diabetes, left ventricular systolic dysfunction, and chronic heart failure. Eur Heart J. 2008;29(10):1224-1240. doi: 10.1093/eurheartj/ehn156

44. Gerstein HC, Bosch J, Dagenais GR, et al. Basal insulin and cardiovascular and other outcomes in dysglycemia. N Engl J Med. 2012;367(4):319-328. doi: 10.1056/NEJMoa1203858

45. Marso SP, McGuire DK, Zinman B, et al. Efficacy and Safety of Degludec versus Glargine in Type 2 Diabetes. N Engl J Med. 2017;377(8):723732. doi: 10.1056/NEJMoa1615692

46. UK Prospective Diabetes Study (UKPDS) Group. Intensive blood-glucose control with sulphonylureas or insulin compared with conventional treatment and risk of complications in patients with type 2 diabetes (UKPDS 33). Lancet. 1998;352(9131):837-853. doi: 10.1016/s0140-6736(98)07019-6

47. ADVANCE Collaborative Group, Patel A, MacMahon S, et al. Intensive blood glucose control and vascular outcomes in patients with type 2 diabetes. N Engl J Med. 2008;358(24):2560-2572. doi: 10.1056/NEJMoa0802987

48. Papanas N, Maltezos E, Mikhailidis DP. Metformin and heart failure: never say never again. Expert Opin Pharmacother. 2012;13(1):1-8. doi: 10.1517/14656566.2012.638283

49. MacDonald MR, Eurich DT, Majumdar SR, et al. Treatment of type 2 diabetes and outcomes in patients with heart failure: a nested case-control study from the U.K. General Practice Research Database. Diabetes Care. 2010;33(6):1213-1218. doi: 10.2337/dc09-2227

50. Boussageon R, Supper I, Bejan-Angoulvant T, et al. Reappraisal of metformin efficacy in the treatment of type 2 diabetes: a meta-analysis of randomised controlled trials. PLoS Med. 2012;9(4):e1001204. doi: 10.1371/journal.pmed.1001204

51. UK Prospective Diabetes Study (UKPDS) Group. Effect of intensive blood-glucose control with metformin on complications in overweight patients with type 2 diabetes (UKPDS 34). Lancet. 1998;352(9131):854-865. doi: 10.1016/s0140-6736(98)07037-8

52. Evans JM, Doney AS, AlZadjali MA, et al. Effect of Metformin on mortality in patients with heart failure and type 2 diabetes mellitus. Am J Cardiol. 2010;106(7):1006-1010. doi: 10.1016/j.amjcard.2010.05.031

53. Andersson C, Olesen JB, Hansen PR, et al. Metformin treatment is associated with a low risk of mortality in diabetic patients with heart failure: a retrospective nationwide cohort study. Diabetologia. 2010;53(12):2546-2553. doi: 10.1007/s00125-010-1906-6
54. Marso SP, Daniels GH, Brown-Frandsen K, et al. Liraglutide and Cardiovascular Outcomes in Type 2 Diabetes. N Engl J Med. 2016:375(4):311-322. doi: 10.1056/NEJMoa1603827

55. Marso SP, Daniels GH, Brown-Frandsen K, et al. Liraglutide and Cardiovascular Outcomes in Type 2 Diabetes. N Engl J Med. 2016;375(4):311-322. doi: 10.1056/NEJMoa1603827

56. Husain M, Bain SC, Mann JFE, et al. No Increased Risk of Heart Failure Hospitalization or Major Cardiovascular Events Observed with Liraglutide in Patients with or without a History of New York Heart Association Class li-lii Heart Failure: Results from the Leader Trial. J Am Coll Cardiol. 2018;71(11):A673. doi: 10.1016/s0735-1097(18)31214-2

57. Zinman B, Wanner C, Lachin JM, et al. Empagliflozin, Cardiovascular Outcomes, and Mortality in Type 2 Diabetes. N Engl J Med. 2015;373(22):2117-2128. doi: 10.1056/NEJMoa1504720

58. Mahaffey KW, Neal B, Perkovic V, et al. Canagliflozin for Primary and Secondary Prevention of Cardiovascular Events: Results From the CANVAS Program (Canagliflozin Cardiovascular Assessment Study). Circulation. 2018;137(4):323-334. doi: 10.1161/CIRCULATIONAHA.117.032038

59. Rahmoune H, Thompson PW, Ward JM, et al. Glucose Transporters in Human Renal Proximal Tubular Cells Isolated From the Urine of Patients With Non-Insulin-Dependent Diabetes. Diabetes. 2005;54(12):3427-3434. doi: 10.2337/diabetes.54.12.3427

60. Heerspink HJ, Perkins BA, Fitchett DH, et al. Sodium Glucose Cotransporter 2 Inhibitors in the Treatment of Diabetes Mellitus: Cardiovascular and Kidney Effects, Potential Mechanisms, and Clinical Applications. Circulation. 2016;134(10):752-772. doi: 10.1161/CIRCULATIONAHA.116.021887

61. Lambers Heerspink HJ, de Zeeuw D, Wie L, et al. Dapagliflozin a glucose-regulating drug with diuretic properties in subjects with type 2 diabetes. Diabetes Obes Metab. 2013;15(9):853-862. doi: 10.1111/dom.12127

62. Chilton R, Tikkanen I, Cannon CP, et al. Effects of empagliflozin on blood pressure and markers of arterial stiffness and vascular resistance in patients with type 2 diabetes. Diabetes Obes Metab. 2015;17(12):1180-1193. doi: 10.1111/dom.12572

63. Fitchett D, Zinman B, Wanner C, et al. Heart failure outcomes with empagliflozin in patients with type 2 diabetes at high cardiovascular risk: results of the EMPA-REG OUTCOME(R) trial. Eur Heart J. 2016;37(19):1526-1534. doi: 10.1093/eurheartj/ehv728

ИНФОРМАЦИЯ ОБ АВТОРАХ [AUTHORS INFO]

*Трухин Иван Викторович [Ivan V. Trukhin]; адрес: 119991, Москва, ул. Трубецкая, д. 8, стр. 2 [address: 8-2 Trubeckaya street, Moscow, 119991 Russian Federation]; ORCID: http://orcid.org/0000-0003-1092-6563; eLibrary SPIN: 1911-9569; e-mail: ivan.trukhin@gmail.com

Петунина Нина Александровна, д.м.н., профессор [Nina A. Petunina, MD, PhD, Professor]; ORCID: http://orcid.org/0000-0001-9390-1200; eLibrary SPIN: 9784-3616; e-mail: napetunina@mail.ru Трухина Любовь Валентиновна, к.M.н. [Liubov V. Trukhina, MD, PhD]; ORCID: http://orcid.org/0000-0001-8997-4984; eLibrary SPIN: 2388-3053; e-mail: Ivtruhina@gmail.com Сизова Жанна Михайловна, д.м.н., профессор [Zhanna M. Sizova, MD, PhD, Professor]; ORCID: http://orcid.org/0000-0002-1241-7074; eLibrary SPIN: 7304-5700; e-mail: sizova-klinfarma@mail.ru Захарова Валерия Леонидовна, к.M.н., доцент [Valeria L. Zakharova, MD, PhD, professor assistant]; ORCID: http://orcid.org/0000-0001-5407-6906; eLibrary SPIN: 3764-6187; e-mail: zkvaleria@mail.ru

\section{ЦИТИРОВАТЬ:}

Петунина Н.А., Трухин И.В., Трухина Л.В., Сизова Ж.М., Захарова В.Л. Сердечная недостаточность и сахарный диабет: взгляд на коморбидность // Сахарный диабет. — 2019. - Т. 22. — №1. - C. 79-87. doi: 10.14341/DM9784

\section{TO CITE THIS ARTICLE:}

Petunina NA, Trukhin IV, Trukhina LV, Sizova ZM, Zakharova VL. Heart failure and diabetes mellitus: insight into comorbidity. Diabetes Mellitus. 2019;22(1):79-87. doi: 10.14341/DM9784 